\title{
Patterns of Lymph Node Metastasis in Differentiated Thyroid Cancer; Clinical Implications with Particular Emphasis on the Emerging Role of Compartment-Oriented Lymph Node Dissection
}

\author{
George H. Sakorafas ${ }^{a}$ Andreas Koureas ${ }^{b}$ Iliana Mpampali ${ }^{a} \quad$ Dimitrios Balalis $^{a}$ \\ Dimitrios Nasikas $^{a}$ Sotirios Ganztzoulas ${ }^{a}$ \\ ${ }^{a}$ Department of Surgical Oncology, Saint Savvas Cancer Hospital, Athens, Greece; \\ ${ }^{\mathrm{b}}$ Department of Radiology, Araiteion Hospital, Athens University, Medical School, Athens, Greece
}

Keywords

Thyroid · Cancer · Metastases · Lymph nodes · Surgery · Lymph node dissection

\begin{abstract}
Recent advances in modern imaging allow an accurate identification of involved or suspicious lymph nodes within the nodal compartments of the neck. Careful ultrasonographic lymphatic mapping of the neck by an experienced radiologist allows a targeted, compartment-oriented (or selective) lymph node dissection to be performed instead of the more extensive modified radical lymph node dissection. Compartment-oriented lymph node dissection necessitates the close cooperation of an experienced radiologist with a particular interest in thyroid and neck ultrasonography. There is emerging evidence that this more conservative strategy allows local control of the disease while at the same time morbidity is minimized and a better cosmetic result is achieved. Further investigation is needed to establish the precise role of this interesting surgical approach.

○ 2019 S. Karger AG, Basel
\end{abstract}

๑) 2019 S. Karger AG, Basel

\section{Introduction}

Lymphatic metastases at the time of diagnosis are common in differentiated thyroid cancer (DTC) [1]. Despite the continuing controversy regarding the role of prophylactic cervical lymph node dissection (CLND) in patients with DTC, it is widely accepted that in the presence of cervical lymphadenopathy CLND is indicated at the time of initial thyroidectomy. Occasionally, skip metastases can occur, but in general lymphatic dissemination occurs in a stepwise fashion [2-5]. Accurate knowledge of these metastatic patterns, in addition to a detailed and reliable preoperative ultrasonographic lymphatic mapping of the neck, will allow a more selective and rational therapeutic approach in patients with DTC and lymph node metastases, thereby avoiding routine extensive dissections and their associated increased morbidity $[6,7]$. The aim of this brief report is to summarize currently available data regarding the patterns of cervical lymph node metastasis in patients with DTC; clinical implications for the practicing surgeon will be briefly discussed, with a particular emphasis on the emerging role of compartment-oriented (so-called 'selective') CLND, based on the findings of preoperative ultrasonographic lymphatic mapping. 
Table 1. Classification of neck lymph nodes -- American Head and Neck Society (2008) Committee for Neck Dissection Classification

Level I Defined superiorly by the body of the mandible, the stylohyoid muscle posteriorly, and the anterior belly of the digastric muscle anteriorly. The 2008 classification revision proposed that, as an alternative to the stylohyoid muscle, the vertical plane defined by the posterior edge of the submandibular gland could be considered as the boundary between levels I and II (see below). Level I may be divided into level Ia (submental), which refers to the nodes in the submental triangle (bound by the anterior bellies of the digastric muscles and the hyoid bone) and Ib (submandibular) which refers to the submandibular triangle nodes.

Level II Related to the upper third of the jugular vein, extending from the base of the skull to the level of the hyoid bone. This level is further subdivided into 2 sublevels, IIb the portion above and behind the spinal accessory nerve, and IIa, the portion anteroinferiorly to this nerve and closer to the internal jugular vein.

Level III Related to the middle third of the internal jugular vein, and are located between the hyoid superiorly and a horizontal plane defined by the cricoid cartilage.

Level IV Related to the lower third of the internal jugular vein, and are located between the cricoid cartilage and the clavicle.

Level V Refers to the lymph nodes located in the posterior triangle of the neck and is bound anteriorly by the posterior border of the sternocleidomastoic muscle and posteriorly by the anterior border of the trapezius muscle. It extends from the convergence of the sternocleidomastoid and trapezius muscle superiorly to the clavicle inferiorly. Level $\mathrm{V}$ is further subdivided by a plane defined by the inferior border of the cricoid cartilage into sublevel Va superiorly (which includes the lymph nodes lying along the spinal accessory nerve) and sublevel $\mathrm{Vb}$ inferiorly (which includes lymph nodes lying along the transverse cervical artery).

Level VI Refers to lymph nodes of the anterior, or central, compartment of the neck, which is defined by the carotid arteries laterally, the hyoid bone superiorly, and the suprasternal notch inferiorly. This level contains the paratracheal nodes, the pretracheal nodes, the parathyryoidal nodes. and the precricoid or Delphian nodes).

Level VII Refers to superior mediastinal lymph nodes.

\section{Classification of Cervical Lymph Nodes}

The neck contains a very rich lymphatic network [8]. Modern classification of neck lymph nodes, based on findings and landmarks from cross-sectional anatomic imaging, is very useful in mapping nodal surgical intervention. The first classification system for neck dissections was published in 1991 by the American Academy of Otolaryngology-Head and Neck Surgery. This schema was later modified (in 2002 and 2008) and has become universally accepted (table 1) [9]. This classification system is composed of 7 major nodal regions (levels or compartments I-VII) and defines a compartment-oriented neck dissection. Sublevel classification is also used when certain zones within the larger levels have independent biologic significance $[6,9]$. The classification of cervical lymph nodes is schematically described in figure 1.

\section{Patterns of Lymph Node Metastasis in DTC and Clinical Implications}

Papillary thyroid carcinoma (PTC) frequently metastasizes to cervical lymph nodes, and a significant percentage of patients (up to $50-60 \%$ or even $90 \%$ for occult metastases) already have lymphatic spread at the time of diagnosis [6]. Current evidence suggests specific patterns of lymphatic metastasis in PTC. Lymph node metastases are commonly observed in the central compartment (level VI). About two-thirds of patients with PTC $>1 \mathrm{~cm}$ will have lymph node metastases in compartment VI, although in only half of these will the metastases be obvious to the naked eye $[5,9]$. Lymphatic metastases are also commonly observed in the lateral compartment (most commonly to levels III and IV) [3, 10-12]. In the study by Noda et al. [13], more than half of the microscopic lymph node metastases were found in the lateral compartment. In about $5-10 \%$ of cases, lateral lymph node metastases may skip the central neck, usually in patients where the tumor is located in the upper poles of the thyroid $[13,14]$. Onoda et al. [15] demonstrated that initial lymph node metastases could occur equally in the central and lateral compartment. Therefore, the central and lateral (levels III, IV, and $\mathrm{VB}$-anterior portion) compartments are frequently involved in PTC, especially in the presence of defined and specific risk factors [13] (see below). Metastases to levels IIA and VB-posterior portion occur at a later stage, while metastases to levels IIB and VA are more rarely observed [11]. Metastases to level I are extremely rare $[11,16]$.

Historically, in the presence of synchronous cervical lymphadenopathy, the standard surgical approach has been the modified radical lymph node dissection at the time of thyroidectomy. This strategy achieves optimal local control of the disease, improves the efficacy of postoperative radioactive therapy, and facilitates patient monitoring during follow-up by measuring serum thyroglobulin. Recent advances in imaging allow, with acceptable accuracy, the recognition of the presence of pathologic lymph nodes within the different compartments [17-19]. High-resolution ultrasonography is being used extensively in the preoperative assessment of cervical lymph nodes (lymphatic mapping; sensitivity $97 \%$, speci- 


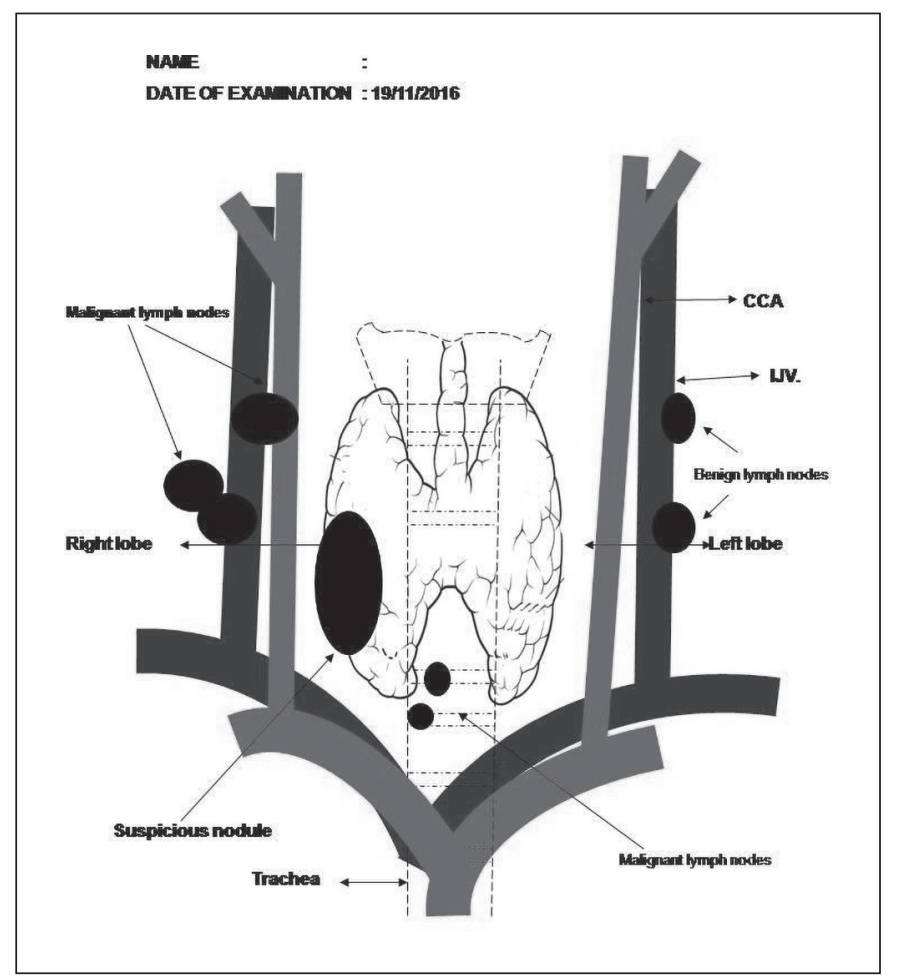

Fig. 1. Schematic representation of lymphatic mapping of the neck in a patient with thyroid cancer. This graphic is based on ultrasonographic findings. We routinely use this schematic lymphatic mapping in patients with thyroid cancer in the operating room (in close collaboration with our experienced radiologist, coauthor no. 2). $\mathrm{CCA}=$ Common carotid artery; IJV = internal jugular vein.

ficity 93\%) [18-21]. Lymphatic mapping can be performed during the initial presentation of the patient with PTC or in the presence of a metachronous recurrence [11]. The accuracy of ultrasonography in recognizing pathologic neck lymph nodes is higher for the lateral nodal compartments, while ultrasonographic evaluation of central compartment lymph nodes is technically more difficult and has a lower sensitivity in detecting metastatic lymph nodes [18, 21, 22]. The experienced radiologist can perform a detailed mapping of abnormal or suspicious lymph nodes within their compartments, which is essential for planning appropriate surgery and adopting a more selective surgical approach (see below). Ultrasound-guided fine-needle aspiration cytology has been proven to be a very useful diagnostic tool with high specificity and accuracy (95-98\% and 95-97\%, respectively) [6]. Neck computed tomography and/or magnetic resonance imaging have a very limited role in preoperative mapping, except in very special indications (such as extensive cervical lymphadenopathy with extension of the diseased thyroid behind the sternum or the clavicle, evaluation for possible involvement by extensive disease of other vital structures of the neck such as the trachea or esophagus, etc.).
Preoperative ultrasonographic lymphatic mapping is indicated in the presence of factors associated with a high probability of lymph node involvement, including increased tumor size (T) (usually $>1 \mathrm{~cm}$ ), suspicion for extrathyroidal extension, clinically evident lymphadenopathy, age $>45$ years, etc. [23, 24].

The ability to perform detailed preoperative ultrasonographic lymphatic mapping generated the idea of a more selective surgical strategy, currently known as 'selective' or 'compartment-oriented' CLND. This strategy is defined as a CLND with removal of less than all of the nodal compartments removed in a typical modified radical CLND [16]; selective CLND lies between the berry picking procedure (currently considered as an unacceptable approach) and the modified radical CLND. Selective CLND has been proposed to reduce the potential morbidity of a more extensive procedure (such as modified radical CLND), without compromising the oncologic outcome [16]. Typical modified radical CLND involves dissection of all levels (I-VI). Based on the above mentioned patterns of lymphatic spread in DTC, level I dissection is not indicated in therapeutic CLND for PTC [16]. Similarly, there are recent reports supporting the avoidance of routine level II and level V dissection when nodes within these levels are ultrasonographically negative $[11,16,25]$. For example, Lim et al. [25] concluded that level V lymphadenectomy may be omitted if positive lymph nodes are not found on frozen section analysis or by ultrasonography in level IV. However, they have proposed level V dissection in the presence of simultaneous multilevel metastases of levels II, III, and IV [25]. Others have proposed the avoidance of routine resection of ultrasonographically negative sublevels IIB and VA, given the rarity of lymph node metastases superior to the spinal accessory nerve, to prevent injury to this nerve [16]. In contrast, level VI is commonly dissected, even as a prophylactic procedure $[10,26]$. Level VI dissection is indicated when there is involvement of lateral compartment(s), even with ultrasonographically negative nodes, due to the high frequency of microscopic metastases in this level (see above) [27]. Finally, since levels III and IV are commonly involved, dissection of these levels is often performed in continuity even if the disease is only evident at 1 of these levels [11]. Although level II dissection is not generally indicated in DTC, dissection of this compartment should strongly be considered in the presence of massive involvement of levels III/IV, even when there is no clinical or ultrasonographic evidence of its involvement [11]. Albeit rare, ultrasonographic evidence of only level II involvement necessitates dissection of levels III, IV, and VI since these levels are commonly involved before the infiltration of level II nodes; however, skip lesions to level II lymph nodes can be observed, but these should be considered as the exception rather than the rule. 
The main concern regarding the role of selective CLND in the management of patients with DTC is oncologic outcome. Nowadays, existing data are few, thereby precluding conclusive recommendations. Welch et al. [16] reported acceptable recurrence rates (8\%), most commonly to lymph nodes in levels III and IV, followed by the central neck (level VI). This compares favorably with recurrence rates following modified radical CLND $(8-12 \%)[16,28,29]$. Recurrences should be treated again selectively, by a compartment-oriented approach, if the level(s) involved has (have) never been dissected [11]. If a recurrence is observed within a previously dissected compartment, then resection of the involved lymph node should be performed [11].

This approach achieves a guided and targeted dissection of carefully selected nodal levels of the neck, thereby avoiding unnecessary and more extensive dissections with their associated morbidity while achieving a better cosmetic result.

The role of the experienced radiologist is crucial in recognizing pathologic lymph nodes and in providing accurate and reliable lymphatic mapping (fig. 1) as a 'guide' for the surgeon during selective CLND.

Morbidity following selective CLND can occur. Complications include mainly neuropathic symptoms due to injury to nerves of the neck (i.e., greater auricular, transverse cervical, and/or supraclavicular nerves of the cervical plexus, spinal accessory nerve, marginal mandibular branch of the facial nerve, cervical sympathetic chain). Welch et al. [16] in their series did not observe chylous drainage following selective CLND; this complication, however, can theoretically occur following dissection of level IV compartment, which contains the thoracic duct. Due to the limited number of studies and lack of statistically appropriate number of patients - especially in the studies examining the role of selective CLND - comparisons between the 2 techniques (classical modified vs. selective CLND) regarding postoperative morbidity are currently impossible. At least theoretically, however, the less extensive tissue dissection and surgical manipulations in selected CLND is expected to be associated with less postoperative morbidity and improved quality of life [30].

In conclusion, existing data are currently scarce, thereby precluding conclusive recommendations. Further investigation in carefully designed clinical trials is needed to establish the precise role of selective CLND in the management of PTC with metastases to the cervical lymph nodes. Based on existing knowledge, however, the following emerging surgical strategy seems reasonable and promising:

A detailed ultrasonographic lymphatic mapping should be performed, ideally by an experienced radiologist. Any compartment with ultrasonographic evidence of metastatic disease should be completely and anatomically dissected.

Central neck dissection is often performed by many surgeons as a prophylactic procedure, especially in the presence of defined risk factors. Central lymph node dissection is indicated when there is involvement of other neck compartments. Morbidity and cosmetic results are in favor of selective CLND. Existing data indicate comparable results from the oncologic point of view, but further investigation in needed, with large numbers of patients and prolonged follow-up to detect potential late recurrences.

\section{Disclosure Statement}

The authors declare no conflicts of interest.

\section{References}

$\checkmark 1$ Haugen BR, Alexander EK, Bible KC, et al.: 2015 American Thyroid Association Management Guidelines for Adult Patients with Thyroid Nodules and Differentiated Thyroid Cancer. The American Thyroid Association Guidelines Task Force on Thyroid Nodules and Differentiated Thyroid Cancer. Thyroid 2016;26:1-133.

-2 Fama F, Cicciu M, Giudice GL, et al.: Pattern of nodal involvement in papillary thyroid cancer. Int J Clin Exp Pathol 2015;8:1162911634.

-3 Machens A, Hinze R, Thomusch O, Dralle H: Pattern of nodal metastasis for primary and reoperative thyroid cancer. World J Surg 2002;26:22-28.
4 Kupferman ME, Patterson M, Mandel SJ, LiVolsi V, Weber RS: Patterns of lateral neck metastasis in papillary thyroid carcinoma. Arch Otolaryngol Head Neck Surg 2004; 130: 857-860.

5 Gimm O, Rath FW, Dralle H: Pattern of lymph node metastases in differentiated thyroid cancer. Br J Surg 1998;85:252-256.

6 Sakorafas GH, Christodoulou S, Lappas C, Safioleas M: Preoperative detection of cervical lymph node metastases in papillary thyroid cancer: a surgical perspective. Onkologie 2009;32:762-766.

7 Sakorafas GH, Sampanis D, Safioleas M: Cervical lymph node dissection in papillary thyroid cancer: current trends, persisting controversies, and unclarified uncertainties. Surg Oncol 2010;19:e57-70.
Grubbs EG, Rich TA, Sturgis EM, et al.: Recent advances in thyroid cancer. Curr Probl Surg 2008;45:156-250.

9 Robbins KT, Shaha AR, Medina JE, et al.: Committee for Neck Dissection Classification, American Head and Neck Society. Consensus statement on the classification and terminology of neck dissection. Arch Otolaryngol Head Neck Surg 2008;134:536-538.

10 Sitges-Serra A, Lorente L, Mateu G, Sancho JL: Central neck dissection; a step forward in the treatment of papillary thyroid cancer. Eur J Endocrinol 2015;173:R199-206.

11 Caron NR, Tan YY, Ogilvie JB, et al.: Selective modified radical neck dissection for papillary thyroid cancer-is level I, II and V dissection always necessary? World J Surg 2006;30:833840. 
12 Wada N, Duh QY, Sugino K, et al.: Lymph node metastasis from 259 papillary thyroid microcarcinomas: frequency, pattern of occurrence and recurrence, and optimal strategy for neck dissection. Ann Surg 2003;237: 399-407.

13 Noda S, Onoda N, Morisaki T, et al.: The significance and the predictive factors of microscopic lymph node metastasis in patients with clinically node negative papillary thyroid cancer: a retrospective cohort study. Int J Surg 2015;20:52-57.

14 Lee YS, Shin SC, Lim YS, et al.: Tumor location-dependent skip lateral cervical lymph node metastasis in papillary thyroid cancer. Head Neck 2014;36:887-891.

15 Onoda N, Ishikawa T, Kawajiri H, et al.: Pattern of initial metastasis in the cervical lymph node from papillary thyroid carcinoma. Surg Today 2013;43:178-184.

-16 Welch K, McHenry CR: Selective lateral compartment neck dissection for thyroid cancer. J Surg Res 2013;184:193-199.

17 Baatenburg de Jong RJ, Rongen RJ, Lameris JS, et al.: Metastatic neck disease. Palpation vs ultrasound examination. Arch Otolaryngol Head Neck Surg 1989;115:689-690.
18 Gonzáez HE, Cruz F, O’Brien A, et al.: Impact of preoperative ultrasonographic staging of the neck in papillary thyroid carcinoma. Arch Otolaryngol Head Neck Surg 2007;133:12581262.

19 Choi JS, Chung WY, Kwak JY, Moon HJ, Kim MJ, Kim E: Staging of papillary thyroid carcinoma with ultrasonography: performance in a large series. Ann Surg Oncol 2011;18:35723578.

20 Ying M, Bhatia KS, Lee YP, Yuen HY, Ahuja AT: Review of ultrasonography of malignant neck nodes: greyscale, Doppler, contrast enhancement and elastography. Cancer Imaging 2013;13:658-669.

21 Kim KE, Kim EK, Yoon JH, Han KH, Moon HJ, Kwak JY: Preoperative prediction of central lymph node metastasis in thyroid papillary microcarcinoma using clinicopathologic and sonographic features. World J Surg 2013; 37:385-360.

22 Kim SS, Lee BJ, Lee JC, et al.: Coexistence of Hashimoto's thyroiditis with papillary thyroid carcinoma: the influence of lymph node metastasis. Head Neck 2011;33:1272-1277.

23 Joo JY, Jin J, Seo ST, et al.: Recurrence in regional lymph nodes after total thyroidectomy and neck dissection in patients with papillary thyroid cancer. Oral Oncol 2015;51:164-169.

24 Thompson AM, Turner RM, Hayen A, et al.: A preoperative nomogram for the prediction of ipsilateral central compartment lymph node metastases in papillary thyroid cancer. Thyroid 2014;24:675-682.
25 Lim YC, Choi EC, Yoon Y-H, Koo BS: Occult lymph node metastases in neck level $\mathrm{V}$ in papillary thyroid carcinoma. Surgery 2010;147: 241-245.

26 Deutschmann MW, Chin-Lenn L, Au J, et al.: Extent of central neck dissection among thyroid cancer surgeons: cross-sectional analysis. Head Neck 2016;38(suppl 1):E328-332.

27 Viola D, Materazzi G, Valerio L, et al.: Prophylactic central compartment lymph node dissection in papillary thyroid carcinoma: clinical implications derived from the first prospective randomized controlled single institution study. J Clin Endocrinol Metab 2015; 100:1316-1324

28 Albuja-Cruz MB, Thorson CM, Allan BJ, et al.: Number of lymph nodes removed during modified radical neck dissection for papillary thyroid cancer does not influence lateral neck recurrence. Surgery 2012;152:1177-1183.

29 O'Neil CJ, Coorough N, Lee JC, et al.: Disease outcomes and nodal recurrence in patients with papillary thyroid cancer and lateral neck nodal metastases. ANZ J Surg 2014;84:240244.

30 Dimov RS: The effect of neck dissection on quality of life in patients with differentiated thyroid cancer. Gland Surg 2013;2:219-226. 